\title{
Endoscopic mucosal resection for gastric epithelial neoplasms: a study of 39 cases with emphasis on the evaluation of specimens and recommendations for optimal pathologic analysis
}

\author{
Gregory Y Lauwers ${ }^{1}$, Shinichi Ban ${ }^{1, *}$, Mari Mino ${ }^{1}$, Shinichi Ota ${ }^{2}$, Takayuki Matsumoto ${ }^{3}$, \\ Shin Arai $^{2}$, Hoi-Hung Chan ${ }^{4,}$, William R Brugge ${ }^{4}$ and Michio Shimizu ${ }^{5}$ \\ ${ }^{1}$ Department of Pathology, Gastrointestinal Pathology Service, Massachusetts General Hospital, Boston, MA, \\ USA; ${ }^{2}$ Third Department of Internal Medicine, Saitama Medical School, Saitama, Japan; ${ }^{3}$ Department of \\ Medicine and Clinical Science, Graduate School of Medical Sciences, Kyushu University, Fukuoka, Japan; \\ ${ }^{4}$ Department of Medicine, Division of Gastroenterology, Massachusetts General Hospital, Boston, MA, USA \\ and ${ }^{5}$ Department of Pathology, Saitama Medical School, Saitama, Japan
}

\begin{abstract}
Endoscopic mucosal resection of gastric neoplasms is a curative technique that avoids surgery and its potential complications. Infrequently performed in the West, the limitations, pitfalls and challenges provided by this new therapeutic modality are not well known by general surgical pathologists. We evaluated a series of 39 endoscopic mucosal resections and assessed the correlation between original biopsies and final diagnoses, depth of excision, status of deep and lateral margins, artifactual changes and recurrence rate. The tumors consisted of 24 intramucosal carcinomas, six high-grade dysplasias, eight low-grade dysplasias and one submucosal invasive carcinoma. The preresection diagnoses corresponded to the final evaluation in $63 \%$ of the cases with previous biopsies. In $37 \%$ of the cases, the biopsies under-diagnosed the neoplasia. The rate of positive margins was $38 \%$. latrogenic changes, that is, intramucosal hemorrhage and electrodiathermic burn, were noted in $44 \%$ of the cases but hindered the pathologic evaluation in only $10 \%$ of the cases. Persistence or recurrence was observed in only seven cases and there was no progression to advanced adenocarcinoma. Based on our experience, we offer some recommendations in order to provide optimal pathologic analysis of endoscopic mucosal resection specimens.
\end{abstract}

Modern Pathology (2004) 17, 2-8, advance online publication, 19 November 2003; doi:10.1038/modpathol.3800012

Keywords: endoscopic mucosal resection; stomach; dysplasia; early gastric cancer; therapy

Independent of the global reduction in the incidence of gastric cancer, the increase in the use of upper endoscopy and better access to medical care have

Correspondence: GY Lauwers, Gastrointestinal Pathology Service, Department of Pathology, Massachusetts General Hospital (WRN 2), 55 Fruit Street, Boston, MA 02114, USA.

E-mail: glauwers@partners.org

${ }^{*}$ Dr S Ban is a Visiting Gastrointestinal Pathology Fellow from the Department of Pathology, Saitama Medical School, Saitama, Japan.

'Dr H-H Chan is a Visiting Gastrointestinal Medicine Fellow from the Division of Gastroenterology, Department of Internal Medicine, Kaohsiung Veterans General Hospital, Kaohsiung, Taiwan.

Received 16 May 2003; revised 18 July 2003; accepted 04 September 2003; published online 19 November 2003 led to a decrease in the size and depth of invasion of gastric cancers. Nowadays, early gastric cancers, that is, adenocarcinomas with invasion limited to the lamina propria or submucosa, independent of lymph node status, represent about 50 and $20 \%$ of all newly diagnosed gastric cancers in Japan and in the West, respectively. ${ }^{1-5}$

Large retrospective series have demonstrated that early gastric cancers have a low risk of lymph node metastasis. For example, intramucosal early gastric cancers presenting with a triad of endoscopic findings including protruding or flat appearance, with nonulcerated surface, and measuring less than $3 \mathrm{~cm}$ rarely show lymphatic extension. ${ }^{6}$ The recognition of these risk factors and the amenability of better endoscopic equipments and techniques, 
including endoscopic ultrasound have resulted in the acceptance of endoscopic mucosal resection as a suitable modality for the treatment of early gastric cancers meeting favorable criteria. Pioneered in Japan in the 1980s, endoscopic mucosal resection is now a routine technique available in most large Japanese medical centers where it is the therapy of choice for $70 \%$ of early gastric cancers. ${ }^{7,8}$ The current guidelines recommend this type of resection for intestinal-type gastric cancers measuring less than $2 \mathrm{~cm}$ and with negative lymph nodes. ${ }^{9}$ Under these guidelines, this technique provides outstanding long-term survival. In a review of 1832 cases reported in 12 series with appropriate follow-up, the survival rate was $99 \%$, with only one death related to metastatic cancer. ${ }^{10}$ In addition, endoscopic mucosal resection is rarely associated with severe complications. Bleeding is reported in about $1.4 \%$ and perforation from 0.5 up to $2.4 \%$ of the resections, respectively. In addition, it offers a superior quality of life compared to gastrectomy. ${ }^{7,10,11}$

In the West, endoscopic mucosal resection of gastrointestinal neoplasms is experiencing a slower development. Several series have reported the experience of few centers with regard to the management of esophageal, rectal and gastric neoplasms. ${ }^{12-14}$ However, considering the risks of gastrectomy (or of esophagectomy and colectomy, for that matter) and its potential negative effect on the quality of life, and given the efficacy of endoscopic mucosal resection for well-selected lesions, it is only a matter of time for Western pathologists to be confronted more routinely with specimens acquired through this technique. Curiously, although the histopathological evaluation of the specimens is crucial since deviation from accepted criteria would determine whether additional surgery is warranted, we are not aware of any series that has focused on the interpretative aspects of gastric endoscopic mucosal resections. Beyond the informative aspect of reviewing this relatively new technique and its indications, our goal in analyzing a series of endoscopic mucosal resections performed for gastric epithelial neoplasms, was to evaluate the specimens from the pathologist's perspective. By focusing on a series of morphologic features that should be analyzed and reported in order to evaluate these resections optimally, we attempted to recognize potential technical pitfalls and to present practical recommendations using the experience of large series as well as ours.

\section{Materials and methods}

In all, 39 gastric endoscopic mucosal resections performed in three institutions (Saitama Medical School and its affiliated hospitals, Massachusetts General Hospital) over a period of 7 years (19932002), and processed according to the protocol described below, were included in this series. The areas to be resected were identified either by magnification endoscopy (seven cases) or chromoendoscopy (32 cases). The 32 patients, 17 males and 15 females, had a median age of 72.5 years (range: 55-94). One patient had three lesions and five patients had two lesions. Four lesions (10\%) were located in the upper one-third of the stomach, 18 lesions $(46 \%)$ were from the middle stomach and 17 $(44 \%)$ of the cases were from the distal stomach. The macroscopic appearance of all lesions was classified as type I (protruded), II (superficial) and III (excavated) according to the classification of the Japanese gastric cancer association (Table 1) ${ }^{15}$ Most were of type IIa (Figure 1a). The endoscopic size of the lesions varied from 3 to $40 \mathrm{~mm}$ (mean: $12.6 \mathrm{~mm}$ ). In 35 cases, the previous preoperative biopsies were available. In 14 of the cases (36\%), an endoscopic ultrasound had been performed preoperatively. The preresection endoscopic ultrasound staging concluded that 10 lesions were limited to the mucosa (Figure 1b) and four extended into the submucosa.

Following a standardized method, after endoscopic evaluation of the size and confines of the lesions, epinephrine and saline (usually 6-15 ml) were injected directly into the submucosa in order to lift the lesions and the surrounding mucosa from the muscularis propria. Then, the abnormal mucosa was grasped by forceps and the resection using coagulation current was performed either with a cap-fitted endoscope into which the lesions would be sucked into while a snare was looped around or the regular snare technique (Figure 1c,d).

Upon reception into the pathology laboratories, all specimens were stretched, pinned down on a firm base and fixed for at least $24 \mathrm{~h}$ in formaldehyde (Figure 1e). After fixation, the specimens were inked and serially sectioned at $2 \mathrm{~mm}$ interval parallel to the longer axis. The sections were sequentially submitted in one or two cassettes, routinely processed and stained with hematoxylin and eosin (Figure 2). The lesions were classified according to recently reviewed criteria of gastric epithelial

Table 1 Macroscopic appearance, location and size of the 39 gastric neoplasms treated by endoscopic mucosal resections

\begin{tabular}{|c|c|c|c|}
\hline & n $(\%)$ & Location & $\begin{array}{l}\text { Average endoscopic } \\
\text { size (range) }\end{array}$ \\
\hline Type I & $10(25.5)$ & $\begin{array}{l}\text { Proximal: } 2 \\
\text { Middle: } 4 \\
\text { Distal: } 4\end{array}$ & $13.4 \mathrm{~mm}(8-25)$ \\
\hline Type IIa & $21(54)$ & $\begin{array}{l}\text { Proximal: } 1 \\
\text { Middle: } 10 \\
\text { Distal: } 10\end{array}$ & $13.9 \mathrm{~mm}(3-40)$ \\
\hline Type IIb & $2(5)$ & Middle: 2 & $5.5 \mathrm{~mm}(4-7)$ \\
\hline Type IIc & $1(2.5)$ & Distal: 1 & NA \\
\hline Type IIa+IIc & $5(13)$ & $\begin{array}{l}\text { Middle: } 2 \\
\text { Distal: } 3\end{array}$ & $8.6 \mathrm{~mm}(3-13)$ \\
\hline
\end{tabular}

Type I, protruded; Type IIa, superficial (elevated subtype); Type IIb, superficial (flat subtype); Type IIc, superficial (depressed subtype). 


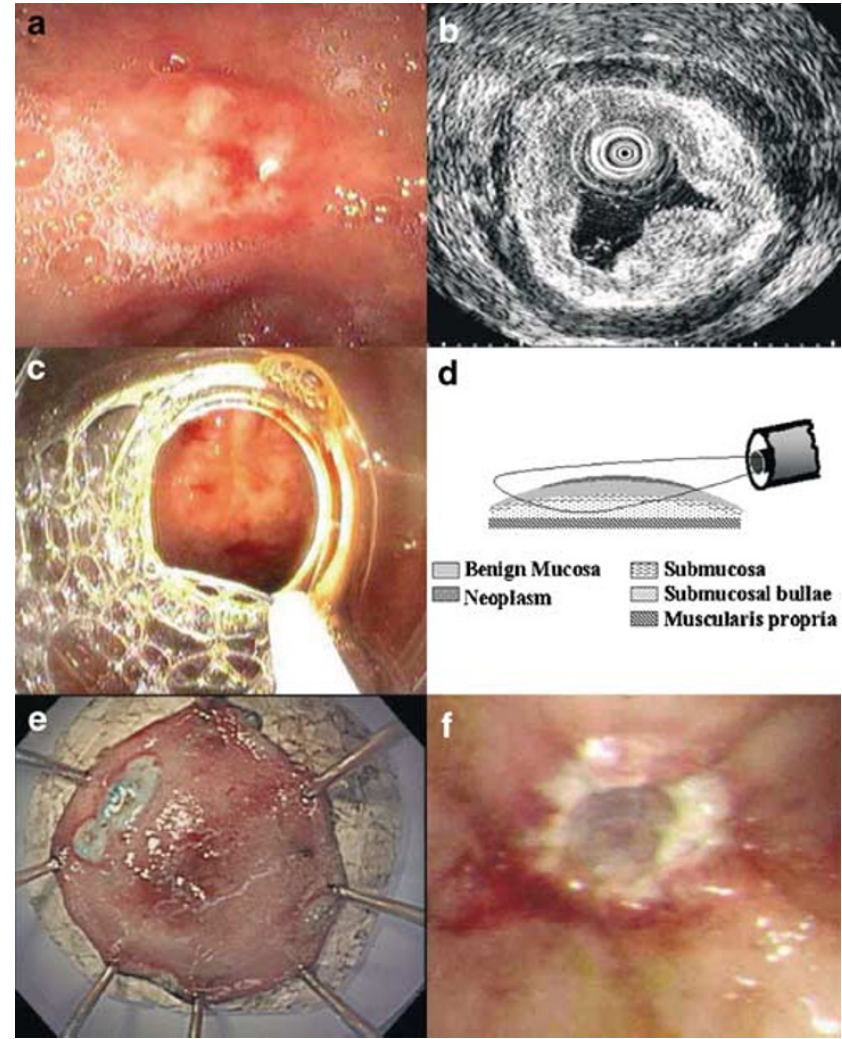

Figure 1 Technical aspects of endoscopic mucosal resection. This type I, polypoid, adenocarcinoma with focal mucosal ulceration (a), appears confined to the mucosal layer on endoscopic ultrasound evaluation (b). (The lesion is represented by a broadbased dome-shaped lesion in the lower aspect of the illustration.) After formation of a submucosal bulla by injection of saline and epinephrine, the lesion is lifted with grasping forceps and a snare is looped around the lesion (c and $\mathbf{d}$ ). After, strangulation of the mucosa, application of electrocautery and resection, the specimen is stretched and pinned on a piece of cork (e). After resection, a large, nonbleeding ulcer is observed on the gastric wall (f).

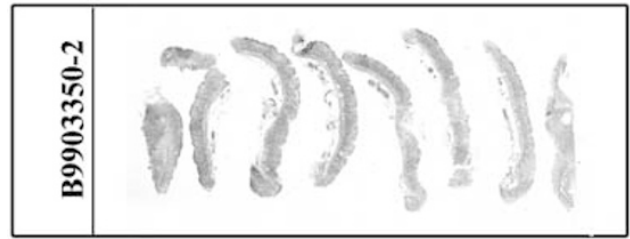

Figure 2 Serial sections of the specimen are sequentially ordered.

dysplasia and carcinoma. ${ }^{16}$ The cases classified as low-grade dysplasia showed limited architectural abnormality with elongated and hyperchromatic nuclei. Nuclear crowding and pseudo-stratification could be seen, but most nuclei remained confined to the basal aspect of the cells. High-grade dysplasia was characterized by more pronounced architectural abnormalities with branching and budding. The nuclei may be vesicular with prominent nucleoli, irregular chromatin and thickened nuclear membrane. Partial or total loss of polarity, as well as high nuclear to cytoplasmic ratio, was frequent. Mitoses

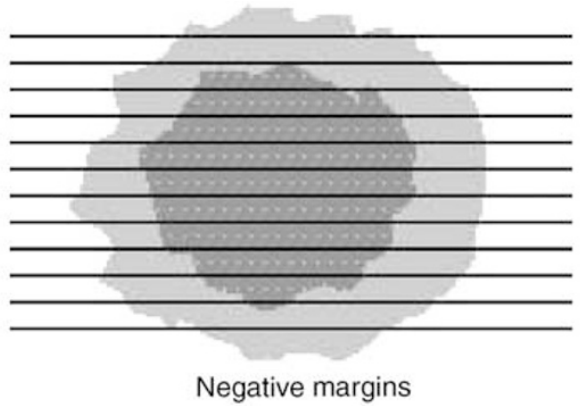

b

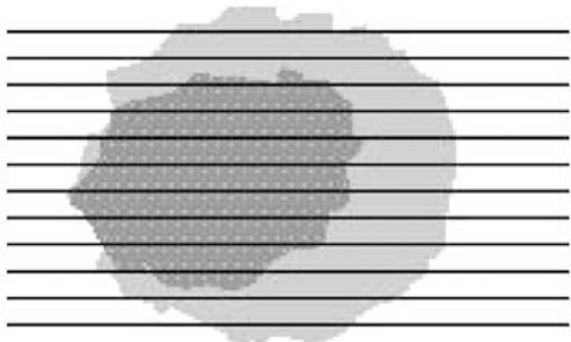

Positive margin

C

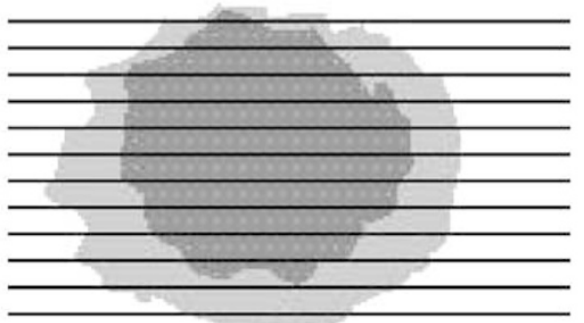

Indefinite marginal status

Benign Mucosa

Neoplasm

Figure 3 Evaluation of endoscopic mucosal resection margins. The specimens are step-sectioned at $2 \mathrm{~mm}$ intervals along the main axis of the specimen as illustrated by the parallel lines. (a) Negative margins with normal mucosa at the periphery (and base). (b) Positive margin(s) is/are recorded when the neoplastic process extends to the lateral (or deep) margins. (c) 'Indefinite marginal status' with neoplastic tissue present on the first en-face section.

were often numerous. The diagnosis of intramucosal carcinoma was made when, in addition to severe cytologic features, evidence of glandular complexity such as, back-to-back glands, syncytial growth pattern, abortive microglands and small clusters of cells could be observed. Invasive carcinoma was diagnosed when a clearly infiltrating growth pattern with desmoplastic stroma was seen. The completeness of resection was evaluated according to criteria previously put forth. ${ }^{17,18}$ A resection was qualified as complete when all margins including deep and lateral were negative (Figure 3a). In addition, the size of the negative margins (clearance) was recorded. A resection was deemed incomplete if the tumor was present either at the inked deep interface 
or peripheral margin(s) (Figure 3b). Importantly, the initial and last en-face slices were to be negative. When the tumor was only present on the en-face sections, the endoscopic mucosal resections were recorded as having an indefinite marginal status since in such cases, the margin could be either positive or with a narrow clearance that could not be evaluated (Figure 3c). A specimen was labeled not evaluable when marked artifacts, either burning or fragmentation beyond possible reconstruction, excluded the assessment of the margins. Finally, we also evaluated the presence of changes that could be attributed to the pre-endoscopic mucosal resection biopsies and more importantly the artifacts secondary to endoscopic mucosal resection and their implications with regard to the evaluation of the specimens.

\section{Results}

The 39 lesions were endoscopically deemed to be completely resected in 22 cases and incompletely in three cases (this information was not recorded by the endoscopists in 14 cases) (Figure 1a). In total, 21 cases were resected en-bloc (54\%). In all, 18 cases were resected in a piece-meal manner. Nine were resected in two fragments, seven in three fragments, one in four fragments and the largest lesion $(40 \mathrm{~mm})$ in 10 fragments.

The resections reached the submucosa in $90 \%$ of the cases $(N=35)$ (Figure 4$)$ and only the muscularis mucosa in four cases $(10 \%)$. No complication, that is, hemorrhage or perforation was recorded. The final pathological diagnosis count included: 24 well-differentiated intramucosal carcinomas with invasion limited to the lamina propria (62\%); six high-grade dysplasias (15\%); eight low-grade dysplasias $(20 \%)$; one case of invasive carcinoma into the submucosa $(3 \%)$. Preresection biopsy-related changes were observed in five cases $(13 \%)$ consisting of focal superficial ulcerations and acute inflammation.

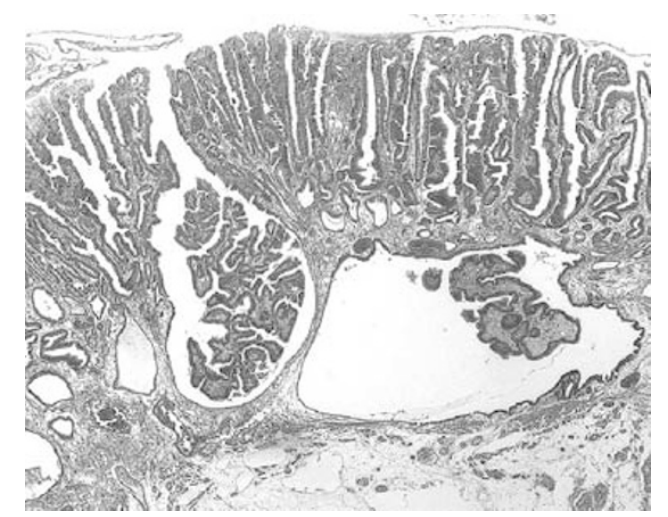

Figure 4 Low magnification of a well-differentiated adenocarcinoma limited to the lamina propria (intramucosal adenocarcinoma). Note that the endoscopic mucosal resection reached the submucosa.
The preresection diagnoses corresponded to the final evaluation in $63 \%$ of the 35 cases with a previous biopsy available. In $37 \%$ of the cases, the biopsy diagnosis under-reported the grade of the neoplasms. The biopsy diagnoses of low-grade dysplasia were confirmed in only eight of 13 cases $(61.5 \%)$ with the final resection diagnoses upgraded either to high-grade dysplasia $(n=4)$ or intramucosal carcinoma $(n=1)$. The original diagnosis of highgrade dysplasia was confirmed in most (seven of nine; $78 \%$ ), while it was upgraded to intramucosal carcinoma in two instances $(22 \%)$. Of the 13 original diagnoses of well-differentiated intramucosal carcinoma 12 were confirmed, with only one case upgraded to invasive (submucosal) carcinoma. The preresection endoscopic ultrasound staging was available in 14 cases. In nine cases (64\%), the endoscopic ultrasound correctly diagnosed an intramucosal neoplasm while it overdiagnosed four cases (submucosal for mucosal invasion) and underdiagnosed only one case (mucosal disease for final submucosal invasion).

Artifacts secondary to endoscopic mucosal resections included intramucosal hemorrhage and electrodiathermic burns. Both were observed in 17 of the cases $(44 \%)$. In most cases the changes were focal and did not hinder the pathologic interpretation. However, these changes were marked and obscured the histologic evaluation including the marginal status in four cases (10\%) (Figure 5a,b).

On routine evaluation, one lateral margin or deep margin was positive in 20 of 37 cases (54\%). In one case, the margins could not be evaluated because of the large number of fragments and there was one case with indefinite marginal status. However, after comparison of the endoscopic and specimen pictures, review of the endoscopists'notes and reconstruction of specimens, the rate of positive margins fell to $46 \%$ (17 cases). In all, 14 cases had one positive lateral margin and one single case had a deep positive margin. Two cases had simultaneously positive deep and lateral margins. The average lateral clearance of the cases with negative margins measured $2 \mathrm{~mm}$ (range: $0.5-5 \mathrm{~mm}$ ), while the average deep clearance measured $0.97 \mathrm{~mm}(0.5-3 \mathrm{~mm})$. Among the cases with available follow-up, five of the 13 cases $(38.5 \%)$ with a positive lateral margin recurred while only one of the 20 cases (5\%) with negative lateral margins had confirmed histologic recurrence. Among the cases with positive lateral margins, the rate of recurrence was $50 \%$ when the positive margin was present on several sections vs $33 \%$ when only one section was involved. Followup was available in only one of the two cases with positive deep and lateral margins (Table 2). Argon plasma coagulation was used in addition to endoscopic mucosal resection in five cases, including two with positive lateral and one with a sole positive deep margin. No evidence of recurrence was noted within 35 months after coagulation. 
6

Endoscopic follow-up with biopsies was not available in seven cases and shorter than a month in two cases (7 and 16 days respectively). In 23 cases, follow-up endoscopic examinations (at 1-35 months; mean 12.9) with biopsy revealed no recurrent disease. This was also the case for the two cases with follow-up biopsies at 7 and 16 days. Seven cases $(18 \%)$ had confirmed histologic persistence or recurrence of disease between 2 and 82 months postendoscopic mucosal resection (mean: 17.6 months) (Table 2). In no case, was there evidence of advanced adenocarcinoma or metastatic disease.

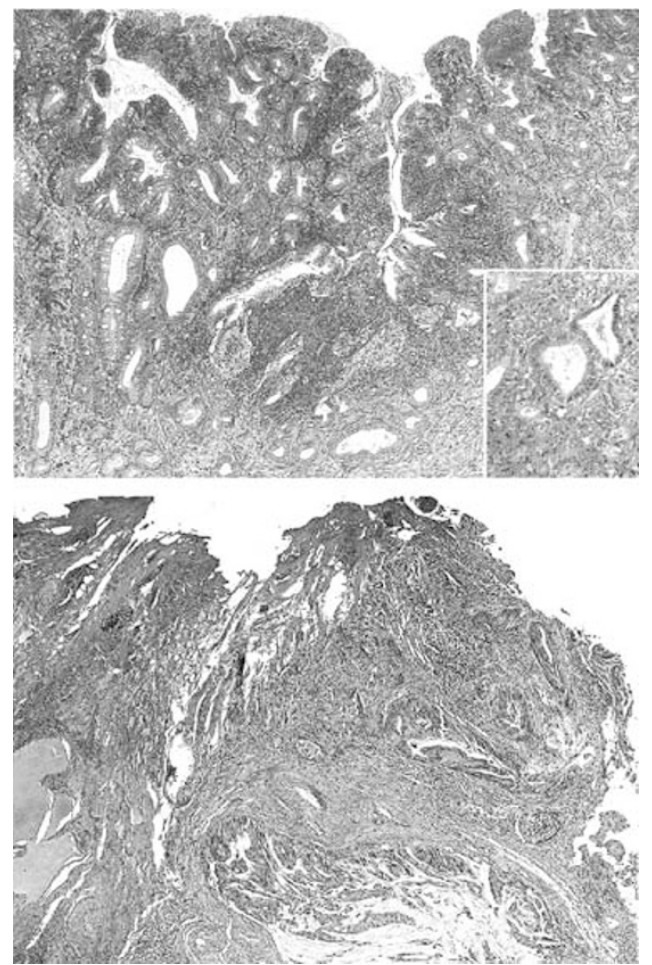

Figure 5 Iatrogenic changes of endoscopic mucosal resection. (a) Massive intramucosal hemorrhage obscuring the lamina propria. Higher power examination shows neoplastic glands surrounded by erythrocytes. (b) Marked electrodiathermic burns can limit the grading of the lesion and sometimes the marginal status as well as the depth of invasion.

\section{Discussion}

At the difference of other endoscopic therapies such as photodynamic and laser therapy, endoscopic mucosal resection is the only endoscopic therapeutic modality providing resected specimens available for pathologic evaluation. In addition, since endoscopic mucosal resections are performed with a curative intent, they should be handled and reported as surgical specimens and therefore, the role of the surgical pathologist is crucial. Appropriate reporting is essential since additional therapeutic options including extension of mucosal resections during a second endoscopic session or recourse to more invasive therapy, that is, surgery, will be directly decided upon the microscopic evaluation of the specimens.

Our intent was not to validate the therapeutic efficacy of endoscopic mucosal resections. Larger studies have reported an overall recurrence rate of about $10 \%(0 \%$ when resection is complete and $35.3 \%$ when the resection was incomplete). ${ }^{7}$ However, our small series with only a few examples of persistent or recurrent disease and no progression to advance adenocarcinoma confirm the validity of endoscopic mucosal resection as an efficient therapeutic option when the indications are strictly chosen. ${ }^{9}$ Severe complications following this endoscopic technique are rare. Tada et $a l^{8}$ reported a rate of $1.6 \%$ for bleeding and $0.06 \%$ for perforation; however, a recent series quoted a perforation rate of $5 \%$, the difference possibly related to the treatment of larger lesions. ${ }^{17}$ Nevertheless, endoscopic mucosal resection should be encouraged in the management of patients who would be at risk for conventional surgery.

The current guidelines for endoscopic mucosal resection of gastric neoplasms include the treatment of well-differentiated mucosal neoplasms of the intestinal type, measuring less than $2 \mathrm{~cm}$ and with no lymph node involvement. ${ }^{9}$ However, some have reported that early gastric cancers with slight invasion into the submucosa measuring less than $5 \mathrm{~mm}$ horizontally were often lymph node negative and therefore could benefit from this modality. ${ }^{19}$ Finally, other investigators advocate even less

Table 2 Clinicopathologic features of the seven persistent/recurrent lesions

\begin{tabular}{llllrlll}
\hline Case & Type/size & Histology & Positive margins & F.U. (mo) & Recurrence & Treatment & F.U. \\
\hline 1 & IIa/10 mm & LGD & Lateral & 82 & LGD & EMR & NA \\
2 & IIa/15 mm & HGD & None & 9 & HGD & EMR & No recurrence at 19 mo \\
3 & IIa/40 mm & IMC & Lateral & 17 & HGD & NA & NA \\
4 & IIa/10 mm & IMC & Lateral & 6 & IMC & NA & NA \\
5 & IIa/10 mm & IMC & Lateral & 4 & HGD & NA & NA \\
6 & IIa/10 mm & IMC & Lateral & 2 & LGD/HGD & PDT & No recurrence at 5 mo \\
7 & I/15 mm & IMC & Lateral, deep & 3 & IMC & EMR & LGD at 2 mo. Treated by PDT and \\
& & & & & & with no recurrence at 14 mo
\end{tabular}

LGD, low-grade dysplasia; HGD, high-grade dysplasia; IMC, intramucosal carcinoma; mo, months; EMR, endoscopic mucosal resection; NA, not available; PDT, photodynamic therapy. 
stringent indications and have reported relative success in well-differentiated intramucosal cancers less than $30 \mathrm{~mm}$ in size, nonulcerated submucosal cancers measuring less than $20 \mathrm{~mm}$ and even poorly differentiated intramucosal carcinomas less than $10 \mathrm{~mm}$ in size. ${ }^{20,21}$ In our own series the median size of the neoplasms $(12.6 \mathrm{~mm})$ fell within the recommended range and still did not preclude the occurrence of recurrence in seven $(18 \%)$ of the cases. Needless to say that given the importance of the proper selection of patients, the pathologic details of the lesions should be under scrutiny. For example, although the determination of size, depth of invasion and lymph node metastasis is critical for the initial selection of patients, it is important to note that endoscopic ultrasound is flawed with significant limitations. The reported accuracy of endoscopic ultrasound in assessing the depth of infiltration of early gastric cancers is between 67 and $72 \% .^{22,23}$ It was $64 \%$ in our own series. It is more accurate for elevated and differentiated neoplasms than depressed and undifferentiated early gastric cancers. ${ }^{22}$ Overall, our results are similar to the series of Yanai et $a l^{23}$ that reported overstaging in about $19 \%$ of the cases, understaging in $2 \%$ and indeterminate assessment in $6.5 \%$ of the cases. These data clearly underline the importance of obtaining an intact specimen if possible and of a meticulous pathologic staging.

Endoscopic mucosal resections have three functions, namely, (a) diagnostic, (b) potentially therapeutic and (c) guidance with regard to determination of further treatment. If the preoperative assessment of the size is confirmed by the pathologic evaluation, four additional pathologic features are critical in the assessment of endoscopic mucosal resection specimens. These are the degree of differentiation, the depth of invasion, the status of the margins (lateral, deep and en-face) as well as clearance of the excision. The status of vascular invasion should also be reported, especially in the cases with submucosal extension. The diagnostic role of endoscopic mucosal resection should not be underestimated. In our series, the original biopsies of these mostly polypoid lesions under-reported the final diagnoses in $37 \%$ of the cases. It is an important shortcoming since the diagnosis of a low-grade dysplasia may lead to the adoption of less stringent follow-up guidelines and leave unchecked lesions with a potential for rapid progression to advanced gastric cancer. ${ }^{16,24}$ It should also be emphasized that endoscopic mucosal resections can be repeated. This is a significant technical advantage given the frequent multifocality of epithelial gastric neoplasms, reaching 19\% in our own series.

Practically, endoscopic mucosal resection specimens should be stretched and mounted on a firm surface (ie, cork or wax block) before fixation. It is a cardinal step since it avoids curling of the specimens' edges during fixation and allows better slicing. Obtaining a picture of the stretched speci- mens is also encouraged, and most particularly when they are fragmented. It may facilitate the reconstruction of the specimens if needed as well as correlation with unexpected findings such as an unpredicted positive margin(s). After fixation, serial sectioning allows optimal determination of the differentiation, depth of invasion, vascular involvement and status of the lateral margins with clearance. The assessment of the clearance is important. In one series, none of the patients with a minimal clearance of $2 \mathrm{~mm}$ developed local recurrence but $16 \%$ of cases with less ample margin recurred..$^{25}$ When adenocarcinoma is present at the margin, recurrence is usually noted in $37-50 \%$ of the cases. ${ }^{17,25,26}$ In our series, the only case with negative margins but recurrence presented with a deep margin clearance of less than $1 \mathrm{~mm}$. Not only the presence of a positive margin but its extension (evaluated by the number of positive sections) is also important. In our series, the rate of recurrence in cases with positive lateral margin rose from 33 to $50 \%$ depending on whether one section or more were involved. As previously pointed out, the therapeutic effect of electrocautery may explain the lower rate of recurrence when the positive margin is limited to one section. ${ }^{27}$ Piecemeal resections are usually difficult to stage accurately. The fragments may be too small for stretching and attempts to reconstruct the specimens may be challenging. In such circumstances, direct communication with the gastroenterologists is cardinal. The comparison of endoscopic pictures and notes with photographies of the specimens received in the laboratory is helpful. The application of different colored inks may also be useful in assessing the overall completeness, or lack thereof, of the resections. One should also keep in mind that artifactual fragmentation of the specimens (manipulation, transport) is not accompanied by electrocautery changes that indicated true mucosal margin. Obviously, the larger the number of fragments, the more difficult is the assessment of the completeness of excision. Technical artifacts such as hemorrhage and electrodiathermic burns $(10 \%$ in our series) can be encountered and warrant to be mentioned in the final report if they limit the histologic interpretation. If significant discrepancies between endoscopic evaluation and pathological findings, such as unexpected positive margin(s) occur, the role of adjuvant treatment either endoscopic or surgical is usually considered. In our series, argon plasma coagulation, second endoscopic mucosal resection and photodynamic therapy have successfully been employed for the treatment of positive margins or recurrence.

We report herein our experience with a series of gastric endoscopic mucosal resections and submit recommendations for the evaluation of specimens (Table 3) based on simple technical steps that we have successfully followed. Given the increasing popularity of endoscopic mucosal resection, 
Table 3 Recommendations for the handling of gastric endoscopic mucosal resection specimens

Measurement of the specimen and lesion and assessment of the surface (ie, ulceration)

Stretching and pinning of the specimen on a firm surface (wood or block of wax)

Photography of the specimen

Fixation for $24 \mathrm{~h}$

Inking of deep and lateral margins

Slicing of the specimen at $2 \mathrm{~mm}$ interval

Submission of the sections in coded cassettes for easy reconstruction of the specimen

pathologists should become acquainted with the technical aspects and limitations of this simple therapeutic modality and be able to recognize the artifacts that may hinder the pathologic evaluation. The analysis of endoscopic mucosal resection specimens cannot be underestimated since it determines the necessity of additional therapy and inappropriate evaluation can lead to flawed postresection decisions and loss of the benefits of this relatively new therapeutic modality.

\section{References}

1 Lewin KJ, Appelman HD. Tumors of the esophagus and stomach. In: Rosai J (ed). Atlas of Tumor Pathology, 3rd series, fascicle 18. Armed Forces Institute of Pathology: Washington, DC, 1996.

2 Everett SM, Axon AT. Early gastric cancer in Europe. Gut 1997;41:142-150.

3 Hisamichi S. Screening for gastric cancer. World J Surg 1989;13:31-37.

4 Sue-Ling HM, Martin I, Griffith J, et al. Early gastric cancer: 46 cases treated in one surgical department. Gut 1992;33:1318-1322.

5 Folli S, Dente M, Dell'Amore D, et al. Early gastric cancer: prognostic factors in 223 patients. Br J Surg 1995;82:952-956.

6 Yamao T, Shirao K, Ono H, et al. Risk factors for lymph node metastasis from intramucosal gastric carcinoma. Cancer 1996;77:602-606.

7 Makuuchi H, Kise Y, Shimada H, et al. Endoscopic mucosal resection for early gastric cancer. Semin Surg Oncol 1999;17:108-116.

8 Tada M, Shimada M, Murakami F, et al. Development of the strip-off biopsy. Gastroenterol Endosc 1984;26: 833-839.

9 Nakajima T. Gastric cancer treatment guidelines in Japan. Gastric Cancer 2002;5:1-5.

10 Kojima T, Parra-Blanco A, Takahashi H, et al. Outcome of endoscopic mucosal resection for early gastric cancer: review of the Japanese literature. Gastrointest Endosc 1998;48:550-554 (discussion 554-555).

11 Takeshita K, Tani M, Inoue $\mathrm{H}$, et al. Endoscopic treatment of early oesophageal or gastric cancer. Gut 1997;40:123-127.

12 Ahmad NA, Kochman ML, Long WB, et al. Efficacy, safety, and clinical outcomes of endoscopic mucosal resection: a study of 101 cases. Gastrointest Endosc 2002;55:390-396.

13 Giovannini M, Bernardini D, Moutardier V, et al. Endoscopic mucosal resection (EMR): results and prognostic factors in 21 patients. Endoscopy 1999;31:698-701.

14 Ell C, May A, Gossner L, et al. Endoscopic mucosal resection of early cancer and high-grade dysplasia in Barrett's esophagus. Gastroenterology 2000;118: 670-677.

15 Japanese Gastric Cancer Association. Japanese classification of gastric carcinoma - 2nd English edition. Gastric Cancer 1998;1:10-24.

16 Lauwers GY, Riddell RH. Gastric epithelial dysplasia. Gut 1999;45:784-790.

17 Ono H, Kondo H, Gotoda T, et al. Endoscopic mucosal resection for treatment of early gastric cancer. Gut 2001;48:225-229.

18 Kondo H, Gotoda T, Ono H, et al. Early gastric cancer: endoscopic mucosal resection. Ann Ital Chir 2001;72:27-31.

19 Ishigami S, Hokita S, Natsugoe S, et al. Carcinomatous infiltration into the submucosa as a predictor of lymph node involvement in early gastric cancer. World J Surg 1998;22:1056-1059 (discussion 1059-1060).

20 Amano Y, Ishihara S, Amano K, et al. An assessment of local curability of endoscopic surgery in early gastric cancer without satisfaction of current therapeutic indications. Endoscopy 1998;30:548-552.

21 Noda M, Kodama T, Atsumi M, et al. Possibilities and limitations of endoscopic resection for early gastric cancer. Endoscopy 1997;29:361-365.

22 Akahoshi K, Chijiiwa Y, Hamada S, et al. Endoscopic ultrasonography: a promising method for assessing the prospects of endoscopic mucosal resection in early gastric cancer. Endoscopy 1997;29:614-619.

23 Yanai H, Tada M, Karita M, et al. Diagnostic utility of 20-megaHertz linear endoscopic ultrasonography in early gastric cancer. Gastrointest Endosc 1996;44: 29-33.

24 Tsukuma H, Mishima T, Oshima A. Prospective study of 'early' gastric cancer. Int J Cancer 1983;31:421-426.

25 Hamada T, Kondo K, Itagaki Y, et al. Endoscopic mucosal resection for early gastric cancer. Nippon Rinsho 1996;54:1292-1297.

26 Mizumoto S, Misumi A, Harada K, et al. Evaluation of endoscopic mucosal resection (EMR) as a curative therapy against early gastric cancer. Nippon Geka Gakkai Zasshi 1992;93:1071-1074.

27 Takekoshi T, Baba Y, Ota H, et al. Endoscopic resection of early gastric carcinoma: results of a retrospective analysis of 308 cases. Endoscopy 1994;26:352-358. 